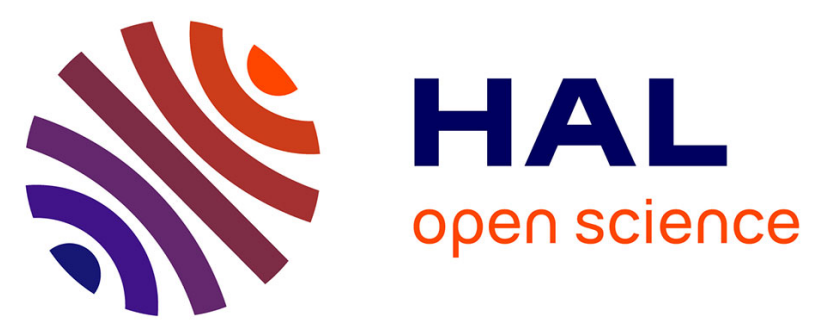

\title{
Modeling Value Evaluation of Semantics Aided Secondary Language Acquisition as Model Driven Knowledge Management
}

Yucong Duan, Christophe Cruz, Abdelrahman Elfaki, Yang Bai, Wencai Du

\section{- To cite this version:}

Yucong Duan, Christophe Cruz, Abdelrahman Elfaki, Yang Bai, Wencai Du. Modeling Value Evaluation of Semantics Aided Secondary Language Acquisition as Model Driven Knowledge Management. 12th IEEE/ACIS International Conference on Computer and Information Science, Sep 2013, Niigata, Japan. pp 259-265, 10.1007/978-3-319-00804-2_20 . hal-00878526

\section{HAL Id: hal-00878526 \\ https://u-bourgogne.hal.science/hal-00878526}

Submitted on 30 Oct 2013

HAL is a multi-disciplinary open access archive for the deposit and dissemination of scientific research documents, whether they are published or not. The documents may come from teaching and research institutions in France or abroad, or from public or private research centers.
L'archive ouverte pluridisciplinaire HAL, est destinée au dépôt et à la diffusion de documents scientifiques de niveau recherche, publiés ou non, émanant des établissements d'enseignement et de recherche français ou étrangers, des laboratoires publics ou privés. 


\title{
Modeling Value Evaluation of Semantics Aided Secondary Language Acquisition as Model Driven Knowledge Management
}

Yucong Duan and Christophe Cruz and Abdelrahman Osman Elfaki and Yang Bai and Wencai $\mathrm{Du}^{*}$

\begin{abstract}
Many theories and solutions have been proposed for the improvement of the learning efficiency of secondary language(L2) learning. However neither an unified view on the functionality of semantics in aiding learning nor an objective measure of the efficiency improvement at theoretical level has been presented in existing literature. This situation hinders the efficient adoption of the semantics aided learning and the explicit planning of a semantics aided learning process. We aim to fill these gaps by adopting an evolutionary strategy towards approaching a holistic solution. Firstly we model the general learning process from cognitive linguistic perspective at the memory level. Then we justify the functionality of semantics aided approach according to specific conditions. Thereafter we propose the quantity measure for the improvement of learning efficiency in terms of reuse level for semantics aided secondary language learning from the perspective of value based analysis.
\end{abstract}

\section{Introduction}

The idea of service computing draws a scenario in which services across various types of barriers such as location, time, culture and languages might be connected to serve users' requirements and fulfill business objectives while guided by economical analysis[1]. However there are many challenges which must be conquered before the idea is fully implemented. For services across multiple language[2] plat-

Yucong Duan and Yang Bai and Wencai Du*

College of information science and technology, Hainan University, China, e-mail: duanyucong@hotmail.com, yang19828@yahoo.com, wencai@hainu.edu.cn

Christophe Cruz

Le2i, CNRS, Dijon, France, e-mail: christophe. cruz@u-bourgogne.fr

Abdelrahman Osman Elfaki

Faculty of Information Sciences and Engineering, MSU, Malaysia, e-mail: abdelrahmanelfaki@gmail.com 
forms to be composed, it requires an agreed understanding and interpretation of the service contracts[3] written in different natural languages. This is a big topic in both natural language processing and involves huge knowledge in linguistics[4]. Many theories and solutions have been proposed. However since most of these theories and solutions originate in various research perspectives and target at various solution scopes, very few unification on the opinions of the mechanism of the linguistic functionality has been achieved among them.

We target at understanding the functionality of the semantics aided approach[5, $6]$ in secondary language(L2) learning[ $[7,8]$ and quantify the measurement of the efficiency improvement. We adopt an evolution perspective towards approaching the targets. Firstly we model the cognitive model of L2 learning in terms of memorization. Secondarily we analyze the functionality and application scopes of the semantics aided approach for L2 learning based on the cognitive model. Finally enlightened by value based analysis[9], we propose contribution calculation formulas for measuring the efficiency improvement [10] after introducing the semantics aided L2 learning in accordance with the degree of reuse. The idea of our proposed evaluation approach might be applied for measurement of a broad scope of knowledge reuse which is not limited to reuse first language (L1) related knowledge for L2 learning specifically.

The rest of the paper is organized as follows: Section 2 presents the solution strategy which crosscuts resolving complexity through model driven approach, introducing value based analysis for measurement from knowledge management perspective, and maintain consistency of semantics through evolving conceptualization. Section 3 presents our research hypotheses which define our targeted problems and restrict our solution framework. Section 4 lists the fundamental research problems and constructs a general cognitive process of learning in terms of memorization. Section 5 formalizes the information foundation of L2 learning and proposes the value quantification formulas for efficiency improvement of L2 learning. Section 6 presents general implementation steps in accordance to the proposed approach. Section 7 presents related work. Section 8 concludes the paper with future directions.

\section{2 discussion on solution strategy}

In this section, we propose the solution strategy of our approach from the dimensions of relieving complexity, quantity analysis and consistency maintenance of expressions.

\subsection{Model driven based strategy on resolving complexity}

Understanding and improve L2 learning[7] processes is a traditional target for cognitive linguistics. Various theories have been proposed and positive results have been 
observed in the past. However most of the confirmed knowledge is limited to certain circumstances under certain hypotheses, so they are partial solutions from a holistic view [11]. We identify that there is an increasingly challenge to manage the complexity of composing related knowledge from a global view in order to harvest their positive contributions while not obligate their application scopes. To composing related L1 related knowledge for the purpose of L2 learning consistently, a premise is that all existing L1 knowledge rules which are to be composed have a complete, clear and uniform description of their application restrictions. However this premise is hardly exist in reality especially in the context of that most of the L1 related knowledge are expressed with informal natural language. From our past experience of resolving complexity through an evolving modeling process in the area of model driven engineering(MDE)[12], we propose to resolve this challenge of complexity through a process of evolving modeling. From an evolutionary perspective of formal semantics $[13,14]$, we propose an analytical framework to support attaining a global level solution based on the model driven process.

\subsection{Value based analysis on knowledge management model}

Value based analysis has been adopted as a fundamental approach in linguistics [4] which is used to justify the motivation of the evolution of languages from a historical perspective [4]. However the concept of value in [4] is too abstract and too general for guiding specific and concrete analysis at service value level [9] which bridges business analysis and project planning. Firstly we plan to model the intended aiding relationships from L1 learning to L2 learning as an knowledge introduction process from a knowledge management $[15,16]$ perspective. At the knowledge management level, we can detail the situations where semantic knowledge contribute positively or negatively. Secondly we abstract the application of the knowledge gained from L1 learning to L2 learning as a reuse process. Then we can extend a formal measure of the efficiency improvement in terms of the degree of the knowledge reuse where both the cost and gains of the knowledge application from L1 to L2 can be measured according to economical formulas.

\subsection{Evolutionary modeling from conceptualization}

- Phenomenon outside: In general, because of the rich phenomena of language learning targets and material, most of existing solutions will be partial solution if they focus on a specific scenario. But every solution can be confirmed as a contribution as long as the theory is consistent and explains the examples. In another word, individual solutions are bundled with specific application scopes: limited age scope, limited culture scope, certain language, language features of noun, verb, adj/adv, grammar, sentence, etc. 
- Phenomenon inside: In natural language expression, there are the phenomena of that multiple semantics might be $\mathrm{M}$ bundled with a single expression or concept [13].

Phenomenon outside reveals challenges of identifying and expressing the application scopes for the purpose of integrating them to construct a whole solution. Phenomenon inside proposes the challenge inside individual expressions if they are not formal. To relieve the disturbance of the understanding of our explanation from these phenomena, we adopt a conceptualization approach [17] to construct an evolutionary explanation framework. In an evolutionary modeling process, the consistency of the semantics of the expression is explicitly maintained while the understanding barriers are supposed to be relieved in the topdown modeling manner where the process of the deepening of the understanding is mitigated from metamodel level to model level.

\section{3 hypotheses on secondary language learning}

L2 learning can benefit from the experience gained through L1 learning for attaining efficiency of both learning behavior/activitiy planning and learning material arrangement.For learning behavior, L2 learning might benefit from the follows:

(i) The best cognitive experience from L1 learning;

(ii) The best cognitive experience for general language learning.

For learning material arrangement at semantic level, L2 learning can take full advantage of reusing the semantics structure which has been constructed among language semantics of $\mathrm{L} 1$ elements.

We believe that from a holistic view learning material arrangement either dominates learning activity/task [18] planning or is independent from it. So in this work, we focus on laying the foundation for learning material arrangement for semantics aided L2 learning. We propose the following hypotheses as the starting point of further discussion for this purpose:

- Hypothesis on basic language elements: the basic notation of a language element and the basic semantics of the same language element are relatively independent existence before the finish of a language learning activity. An obvious fact is that we know basic semantics even before we learn the notations to express them. These semantics have their relatively independent existence.

- Hypothesis on the form of semantics: based on the hypothesis of the independent existence of language semantics, we make a further hypothesis that during the first language learning process language elements which are learned by us form several semantic net or semantic structures (SSN) [19]. In this paper, at general knowledge management level, we do not distinguishing syntactic structure and semantic structure in the process of modeling general semantics as to be managed knowledge pieces. 
- Hypothesis on the applicability of reuse: at learning material preparation level, there are overlaps among the SSN(L1) of attained L1 material and the SSN(L2) to be formed in targeted L2 learning material.

- Hypothesis on the efficiency of reuse: Limited to a simplified learning activity cost analysis, reusing SSN(L1) will be cost efficient for learning L2 since that it saves the behavioral effort [7] of constructing SSN(L2).

\section{Modeling the general learning process}

We present the fundamental questions which any related research can not avoid and model the general learning process as a basis for further work.

\subsection{Fundamental questions}

Not like empirically employing semantics gained from L1 learning directly as means of improving efficiency of L2 learning, we need to understand the following questions before designing a guided application of semantics aided L2 learning:

- What is the function of semantics in language learning?

- How could its functionality be justified as positive and with what restrictions?

- How could an efficient L2 learning be planed based on knowledge gained from L1 learning?

To answer these questions and ensure the understanding of the explanation can be rendered uniformly to the audiences with clear semantics bundled to intended concepts, we adopt a conceptualization based evolutionary modeling approach in the following sections.

\subsection{Modeling the general learning from cognitive perspective}

To make things simple, a semantic aided L2 learning process can be modeled as confirming inheritably to the general rules of a general semantic aided learning. In a general semantic aided learning, semantic of a language symbol is any intended meaning which is bundled with that symbol.

Hypothesis on learning through memorization: general language learning is a process of memorization of either static language material or dynamic language production mechanism which turns previously unknown or outside language material or grammar into known or usable language material or grammar inside the mind of a learner. We model the progress of the learning process as with consecutive progressing stages from the perspective of memorization as follows. 
1. Memorization of language symbols - During this stage, we learn the symbols which is used for representing semantics at existence level for our communication sensor organs such as vision by eyes or speaking/hearing by month/ear, etc.

2. Memorization of the integration of language symbol + basic semantics of the symbol - During this stage, we relate our inner semantics knowledge with language symbols to form common concepts which are shared by people in the society.

feasibility of consistency vs. representation (symbol composition): if it is required that a single semantic must be assigned with a unique composition of language symbols, we can imagine that simply from the perspective of learning burden of huge language symbol composition it is not feasible.

3. Memorization of language symbol + extensive semantics of the symbol - During this stage, we learn to relate more semantics to basic concepts.

Multiple semantics formation (concept composition): We model this as an evolutionary process of relating semantics of our expressions composing existing concepts. This stage help us to realize concepts enrichment while avoids the obsession of huge increase of amount of semantic symbols.

4. Memorization of language elemental concept/word + grammar - Language elemental concept/word: the integration of language symbol + semantics.

During this stage, we start to retrospect on the composition of concepts and identified grammar which is the common order of constructing an expression. Grammar helps to unify the expression of semantics while it does not interfere the intended semantic content.

5. Memorization of language elements emerging from language expressions - During this stage, we start a journey of language learning based on thought which is on top of existing language mechanism itself. We identify meanings of language expressions as new concepts. This process constitutes an evolution mechanism of a language itself from elementary to higher level. This stage differs from previous stages in the following aspect: observation is changed from "semantic $\rightarrow$ concept" to "expression $\rightarrow$ semantic". During the first 4 stages, our observation relies on the mode of bundling new semantics with language labels to form language elements. In this stage, new semantics is observed from language expressions as a whole. The reasoning process is also supported by our existing language knowledge..

\section{Modeling and evaluation on learning efficiency change}

\subsection{Modeling and conceptualization on multiple semantics}

If a language (LN) is composed as a sequence of M language elements(LE) and the grammar (GR) of it:

$$
L N(x)=\operatorname{set}(L E)+G R
$$


If a language expression(LEP) is composed as a sequence of $M>=2$ pieces of LEs following an order:

$$
L E P(x)=L E(1) \rightarrow L E(2) \rightarrow \ldots \rightarrow L E(M)
$$

For every $\operatorname{LE}(\mathrm{y})$, the amount of possible semantics which are bundled with it is denoted as pse(y). We denote the possible semantics which are bundled with a $\mathrm{LEP}(\mathrm{x})$ as:

pse $1, p s e 2, p s e 3, \ldots, p s e N$.

Ideally the amount of the possible integrated semantics of LEP(x) is a Descartes multiply expression:

$$
p s e(\operatorname{LEP}(x))=p s e(1) * p s e(2) * \ldots * p s e(m)
$$

From a conceptualization perspective, we can observe that there is an implicit transfer that a LEP expression can become a language element in the form of LEs recursively. A notable thing is that after the connection which forms an expression chain, the semantics of LEP(x) relies on the relationship of composing LEs which is different from the beginning stages where semantics relies solely on the set on of composing LEs and the grammar. We denote this transfer of the essence of the semantics as a transfer from entity to relationship[20] :

$$
\text { ENTITY } \rightarrow \text { RELATIONSHIP. }
$$

If we consider the conceptualization process of an elementary LE as an explicit relationship, it can be reached that at semantics level semantic is relationship:

SEMANTIC $=$ RELATIONSHIP

A formal clarification on this formula is as follows:

An explicit explanation from the perspective of the existence of multiple semantics for a single concept [13] is there are more than one semantic bundled with concept(SEMANTIC), concept(RELATIONSHIP) and concept(ENTITY). If we denote a notation of "=" as a binary equity relationship among two concepts of concept(A) and concept(B) iff there is " $n>=1$ " times of shared semantics among the bundled semantic sets of two concepts of concept(A) and concept(B). If we denote a notation of "! = " as a binary equity relationship among two concepts of concept(A) and concept(B) iff there is " $n>=0$ " times of not shared semantics among the bundled semantic sets of two concepts of concept(A) and concept(B). Then the following formulas are founded in the complete background of $<$ entity, relationship $>$ following a description grammar called natural expression function(NEF) [21]:

For concept(SEMANTIC):

$$
\begin{aligned}
& \text { concept }(\text { SEMANTIC })=\text { concept }(\text { RELATIONSHIP }) \\
& \text { concept }(\text { SEMANTIC })=\text { concept }(\text { ENTITY }) \\
& \text { ( if we consider concept(CONCEPT) is a form of entity }) \\
& \text { For concept(RELATIONSHIP): } \\
& \text { concept }(\text { SEMANTIC })=\text { concept }(\text { RELATIONSHIP }) \\
& \text { concept }(\text { RELATIONSHIP }) !=\text { concept }(\text { ENTITY })
\end{aligned}
$$

(if we refer the distinction of relationship vs. entity as a rule from a higher model level)

From the complete comparison background of $<$ entity, relationship $\rangle$, we can conclude from above at semantic level: 
concept $($ SEMANTIC $)=$ concept $($ RELATIONSHIP $)$ for semantics understanding; and concept $(S E M A N T I C) !=$ concept $(R E L A T I O N S H I P)$ for conceptual level distinguishing of the two concepts as a whole.

In this paper, when mentioning semantics of semantics aided language learning we refer by default the semantics of a LEP which contains more than $1 \mathrm{LE}$.

\subsection{Justify the activity of semantics based approaches}

We try to answer how in general a learning efficiency improvement can be guaranteed as positive at semantic level?

Hypothesis on linked LEs: the learning effort for a set of individual language elements is higher than the learning effort for the same set language elements which are however interlinked with meaningful/pre-attained knowledge structures. It is formalized as follows:

$$
L E_{\{\text {individual } L E\}}<L E_{\text {linkedLEs }}
$$

In general, semantics aided language learning (SAL) is based on reusing existing knowledge and experience to save the effort of learning language elements.

Knowledge reuse: reuse the static structure in knowledge store which matches the structure of linked language elements.

Experience reuse: reuse the experience of knowledge learning from L1 learning, such as similar words, similar categories, and similar grammar, etc.

\subsection{Value based cost modeling at the level of reuse}

Here we try to model how an efficient learning process can be implemented at knowledge management level. Some of the semantics of individual symbols can be related according to some existing knowledge or existing experiences. According to our hypotheses at Section 3, the explicitly reuse of the existing knowledge from L1 learning will greatly save the learning effort of L2.

We propose to measure the efficiency improvement of L2 learning in terms of value with a demonstrative formula of cost vs. gain calculation as follows:

$I M E=L E-R N * E R E$

IME:improvement of learning efficiency

LE: gross learning effort which are spent by a learner without help

$\mathrm{RN}$ : times of reuse

ERE: effort of learning the reused knowledge without help.

Derived conclusion:

As long as ERE is positive and $R N>0$, IME is positive. 


\subsection{Analyzing L2 learning according to cognitive model}

Here we compare the learning process of L1 vs. L2 referring to the 5 stages of the general cognitive model. For L1 learning, all of the 5 stages of the general model can apply. For L2 learning, the difference might depend on the specific approach which are adopted by a L2 learning. Anyway the efficiency of the L2 learning will match either the situation of a form of L1 learning or L2 learning. We can expect the efficiency of L2 learning be no less than that of a L1 learning process normally based on the formula of:

$I M E=L E-R N * E R E$.

Under the direction of SAL, we can plan a L2 learning process corresponding to the 5 stages of the general learning model as follows:

For stage 1, the basic notation memorization process of a L2 will be similar to a L1 if there is no linguistic knowledge in which the L1 and L2 can be related to a language family.

For stage 2 and 3, the semantic observation of L2 is by way of semantics of L1 instead of from the result of direct observation with individual senses of a learner.

For stage 4 , there are a lot of knowledge which can be reused according to different types of semantic knowledge of word classes. However sometimes there are side-effects ranging from the mismatch of semantics of seemingly similar concepts to inconsistency of seemingly consistent grammar rules[22]. In general, special attention need to be paid to abnormal situations during the process of enjoying reusable knowledge from L1.

For stage 5, a L2 learning has the advantage of that it can benefit from not only its accumulated language learning capability of L2 but also the learning capability of L1. Before the level of a learner's L2 surpass his/her level of L2, he/she has the potential to benefit from the positive difference between his/her level of L1 and his/her level of L2 by using his/her learning capability of L1 to learn L2. The restrictions of usage can be modeled as:

level $(L 1)-\operatorname{level}(L 2)>0$

This restriction is at general level. A specific condition for guiding implementation is that as long as a semantic which is among the possible semantics of a LE in L1 does not exist in the learned capability of a L2 for L2 learner, he/she has the possibility to benefit from using his/her L1knowledge to understand the target language elements of his/her L2. The restriction at semantic level can be modeled as:

$$
\text { pse }(\operatorname{LEP}(x))_{L 1}>\operatorname{pse}(\operatorname{LEP}(x))_{L 2}
$$

\section{6 stages of a general implementation}

Based on our previous analysis, we propose the main stages for a SAL learning process consequentially. 
1. Cognitive model. It describes the underlying process of how human learn a secondary language: the order of learning; the characters of the learning target, the evolution of learning from simple to complex, etc.

2. Conceptual model. A conceptual model is based on a conceptualization process. It will set the concept architecture for standardizing the description of the processing and the organization of the learning material in terms of linguistic discipline.The conceptualization process of L2 learning will be different from L1 learning process.

3. Psychological model This model is optional but can be adopted for the improving the efficiency of a comprehensive learning by following human learning psychological rules.

4. Learning model/architecture. This architecture uses the conceptual model to describe learning strategies which confirm to the facts contained in the cognitive model and psychological model.

5. Computation model. Algorithms will be designed and implemented towards goals of information processing in the form of knowledge management or efficiency evaluation according to above architecture with concrete conditions.

6. Experiments and modification. Find test cases and use the feedback to refine the proposed theories.

In this paper, we focus on construct the theoretical foundation for the implementation of these stages.

\section{7 related work}

Secondary language acquisition approaches can be classified according to many categories[23]. From the category of the types of learning targets, most of current existing learning strategies target at learning at vocabulary level [8]. Our proposed approach can be applied not only to vocabulary level but also syntactic level and semantic level. From the category of the types of learning knowledge, most of existing approaches are either based on empirical experiences or on cognitive behavioral models [8]. Since these strategies are mostly based on directly observation or experimental data[24], it is very difficult to extend them into more complex theory to guide more complex targets at semantics level. Also since the scenarios where these approaches are derived are difficult to be related, it is a big challenge to compose related approaches. Our proposed approach supports L1 learning knowledge identification based on reusable pattern identification and knowledge composition centering reuse activity planning. It also supports abstract validation and evaluation in terms of reuse level improvement before experimental test which might be influenced by unknown factors in specific context. Tao et al.[16] emphasis the importance of promoting semantic level learning by ways of knowledge management in E-learning, however the work does neither justify the rationality of introducing semantics nor provides a quantity measure. 
- On cognitive hypothesis: Cummins [7] has proposed a hypothesis on L2 learning as the common underlying proficiency(CUP). CUP presents that the conceptual knowledge which is developed in L1 learning will contribute to L2 learning as that it can be reused without the need to develop it again. Our work develops a systemic analysis on this view and details related states in a cognitive learning model..

- On evaluation model : Saussure [4] proposed that there is underlying value difference which drives the evolution of languages. We focus on the value evaluation representing the efficiency of L2 learning and details the prototype of the calculation formulas which can be extended for modeling general problems in linguistics..

\section{Conclusion and future directions}

Many challenges in information technology can be attributed to fundamental problems at linguistics level. From an evolutionary perspective, we believe that understanding the L2 mechanism is part of the key start point towards building an ideal solution for across language platform information processing such as automatic processing of service contracts in an international background. In view of the absence of theories covering fundamental issues of semantics aided L2 learning at semantic level, we propose a systemic solution which models the cognitive learning process at knowledge management level, introduces quantity analysis of the efficiency improvement at the economical level from the perspective of reuse. The significance of this work lies mainly in that we try to answer the fundamental questions in L2 learning by transferring these problems into other domains where there are familiar solutions through modeling. Specifically we contribute in the following points:

- formalizing information foundation of semantics aided L2 learning : from a conceptualization approach [17], we formalize the information foundation of applying semantic aided L2 learning as overlap between SSN(L1) and SSN(L2).

- modeling L2 learning for value based analysis: by way of modeling the L2 learning process as a process of knowledge introduction of knowledge management[15] from L1 learning to L2 learning, we successfully transfer an abstract learning process into a domain where value based analysis can be applied directly.

- quantification of learning efficiency improvement : we identify the degree of reuse as a key variables/factors for formulating the calculation/measure of the quantity of learning efficiency improvement. We propose demonstrative formulas for applying in L2 learning.

We plan to proceed towards learning activity planning, and refine the proposed approach with comparative experiment[25]. We would also like to use our evaluate approach to identify the cons and pros of existing practices from many sources including empirical and cognitive psychological sources and incorporate them to construct personalized [26] solution. 


\section{Acknowledgment}

This paper was supported in part by China National Science Foundation grant 61162010 and by Hainan University Research program grant KYQD1242. * stands for corresponding author.

\section{References}

1. Duan, Y.: A Survey on Service Contract. In: SNPD. (2012) 805-810

2. Comerio, M., Truong, H.L., Paoli, F.D., Dustdar, S.: Evaluating Contract Compatibility for Service Composition in the $\mathrm{SeCO}_{2}$ Framework. In: ICSOC/ServiceWave. (2009) 221-236

3. Duan, Y.: Service Contracts: Current state and Future Directionsmeasure. In: ICWS. (2012) 664-665

4. de Saussure, F.: Course in General Linguistics. Open Court, Chicago, IL (1998)

5. Dunlap, S., Perfetti, C.A., Liu, Y., Wu, S.: (Learning vocabulary in chinese as a foreign language: Effects of explicit instruction and semantic cue reliability)

6. Ellis, N.C.: At the interface: Dynamic interactions of explicit and implicit language knowledge. Studies in Second Language Acquisition 27 (2005) 305-352

7. Cummins, J.: Language, Power and Pedagogy: Bilingual Children in the Crossfire. Multilingual Matters, Clevedon (2000)

8. O’Malley, J., Chamot, A.: Learning Strategies in Second Language Acquisition. The Cambridge Applied Linguistics Series. Cambridge University Press (1990)

9. Duan, Y.: Modeling Service Value Transfer beyond Normalization. In: SNPD. (2012) 811-816

10. Norris, J.M., Ortega, L.: Effectiveness of 12 instruction: A research synthesis and quantitative Meta-Analysis. Language Learning 50(3) (2000) 417-528

11. Duan, Y., Lee, R.: Knowledge Management for Model Driven Data Cleaning of Very Large Database. In: SCI(443)). (2012) 143-158

12. Duan, Y., Cheung, S.C., Fu, X., Gu, Y.: A Metamodel Based Model Transformation Approach. In: SERA. (2005) 184-191

13. Duan, Y.: Semantics Computation: Towards Identifying Answers from Problem Expressions. SSNE 2011 (2011) 19-24

14. Duan, Y.: A Dualism Based Semantics Formalization Mechanism for Model Driven Engineering. IJSSCI 1(4) (2009) 90-110

15. Duan, Y., Cruz, C., Nicolle, C.: Architectural Reconstruction of 3D Building Objects through Semantic Knowledge Management. In: SNPD. (2010) 261-266

16. Tao, F., Millard, D.E., Woukeu, A., Davis, H.C.: Managing the semantic aspects of learning using the knowledge life cycle. In: ICALT. (2005) 575-579

17. Duan, Y., Cruz, C.: Formalizing Semantic of Natural Language through Conceptualization from Existence. IJIMT 2(1) (2011) 37-42

18. Swain, M., Lapkin, S.: Task-based Second Language Learning: The Uses of the First Language. Arnold (2000)

19. Meng, H.M., Siu, K.C.: Semiautomatic acquisition of semantic structures for understanding domain-specific natural language queries. IEEE Trans. Knowl. Data Eng. 14(1) (2002) 172181

20. Chen, P.P.: The entity-relationship model - toward a unified view of data. ACM Trans. Database Syst. 1(1) (1976) 9-36

21. Duan, Y., Kattepur, A., Zhou, H., Chang, Y., Huang, M., Du, W.: Service value broker patterns: Towards the foundation. In: ICIS. (2013)

22. Corder, S.: The significance of learner's errors. International Review of Applied Linguistics in Language Teaching 5(4) (1967) 161-170 
Evaluation Semantics Aided Secondary Language Acquisition

23. Stockwell, G.: A review of technology choice for teaching language skills and areas in the call literature. ReCALL 19(2) (2007) 105-120

24. Dearman, D., Truong, K.: Evaluating the implicit acquisition of second language vocabulary using a live wallpaper. In: ACM HFCS. (2012) 1391-1400

25. Allum, P.: Call and the classroom: the case for comparative research. ReCALL 14(1) (2002) $146-166$

26. Ayala, G., Paredes, R.G., Castillo, S.: Computational models for mobile and ubiquitous second language learning. Int. J. Mob. Learn. Organ. 4(2) (2010) 192-213 